Pacific

Journal of

Mathematics

\title{
GENERALIZED HANDLEBODY SETS AND NON-HAKEN
} 3-MANIFOLDS

\author{
Jesse EdWard Johnson AND Terk Patel
}




\title{
GENERALIZED HANDLEBODY SETS AND NON-HAKEN 3-MANIFOLDS
}

\author{
JESSE EDWARD JOHNSON AND TERK PATEL
}

\begin{abstract}
In the curve complex for a surface, a handlebody set is the set of loops that bound properly embedded disks in a given handlebody bounded by the surface. A boundary set is the set of nonseparating loops in the curve complex that bound two-sided, properly embedded surfaces. For a Heegaard splitting, the distance between the boundary sets of the handlebodies is zero if and only if the ambient manifold contains a nonseparating, two sided incompressible surface. We show that every vertex in the curve complex is within two edges of a point in the boundary set.
\end{abstract}

\section{Introduction}

The curve complex $C(\Sigma)$ for a compact, connected, closed, orientable surface $\Sigma$ is the simplicial complex whose vertices are loops (isotopy classes of essential, simple closed curves) in $\Sigma$ and whose simplices correspond to sets of pairwise disjoint loops in $\Sigma$. Given a handlebody $H$ and a homeomorphism $\phi: \Sigma \rightarrow \partial H$, we can define the following subsets of $C(\Sigma)$.

The handlebody set $\mathbf{H}$ is the set of loops that bound properly embedded (essential) disks in $H$. The genus $g$ boundary set $\mathbf{H}^{g}$ is the set of nonseparating loops such that each bounds a properly embedded, two-sided, incompressible, genus- $g$ surface in $H$. Note that $\mathbf{H}^{0}$ is a proper subset of $\mathbf{H}$, specifically the set of all the nonseparating loops in $\mathbf{H}$. Define the boundary set to be the union

$$
\mathbf{H}^{\infty}=\bigcup_{g \geq 0} \mathbf{H}^{g} .
$$

We will say that a set $A$ of vertices in $C(\Sigma)$ is $k$-dense if every vertex in $C(\Sigma)$ is within $k$ edges of a point in $A$.

Theorem 1. If $\Sigma$ has genus 3 or greater, then $\mathbf{H}^{\infty}$ is 2-dense in $C(\Sigma)$.

MSC2000: 57M50.

Keywords: curve complex, non-Haken 3-manifold.

Research supported by NSF MSPRF grant 0602368. 
The proof presented here does not work for genus two surfaces. However, Schleimer has shown in [2005] that the orbit of a vertex of $C(\Sigma)$ under the action of the Torelli group is 5-dense. This implies that for a genus two handlebody, $\mathbf{H}^{\infty}$ is $n$-dense for some $n \leq 5$.

In contrast to $\mathbf{H}^{\infty}$, a fixed genus boundary set $\mathbf{H}^{g}$ has a geometric structure much closer to $\mathbf{H}$, which is not $k$-dense for any $k$. This is demonstrated by the following two Lemmas, the first of which is a corollary of Conclusion III.15 in [Jaco 1980] and the second of which follows from a Theorem of Scharlemann [2006].

Lemma 2. If $\Sigma$ has genus three or greater and $v \in \mathbf{H}$ then

$$
d\left(v, \mathbf{H}^{g}\right)=1
$$

for every $g>0$. If $\Sigma$ has genus two then

$$
d\left(v, \mathbf{H}^{g}\right)>1
$$

for every $g$.

Lemma 3. For $g \geq 1$, the set $\mathbf{H}^{g}$ is disjoint from $\mathbf{H}$ and contained in a $2 g$ neighborhood of $\mathbf{H}$.

For this paper, every 3-manifold will be compact, connected, closed and orientable. A Heegaard splitting for such a 3-manifold $M$ is a triple $\left(\Sigma, H_{1}, H_{2}\right)$ where $\Sigma \subset M$ is a compact, connected, closed, orientable surface and $H_{1}, H_{2} \subset M$ are handlebodies such that

$$
\partial H_{1}=\Sigma=\partial H_{2} \quad \text { and } \quad M=H_{1} \cup H_{2} .
$$

The inclusion maps from $\partial H_{1}$ and $\partial H_{2}$ onto $\Sigma$ determine handlebody sets $\mathbf{H}_{1}$ and $\mathbf{H}_{2}$, respectively. The distance of the Heegaard splitting, as defined by Hempel in [2001] is the distance $d(\Sigma)=d\left(\mathbf{H}_{1}, \mathbf{H}_{2}\right)$ between the two handlebody sets.

The inclusion maps also determine boundary sets $\mathbf{H}_{1}^{g}, \mathbf{H}_{2}^{h}, \mathbf{H}_{1}^{\infty}, \mathbf{H}_{2}^{\infty}$, allowing us to generalize this distance to the $(g, h)$-distance

$$
d^{g, h}(\Sigma)=d\left(\mathbf{H}_{1}^{g}, \mathbf{H}_{2}^{h}\right)
$$

and the boundary distance

$$
d^{\infty}(\Sigma)=d\left(\mathbf{H}_{1}^{\infty}, \mathbf{H}_{2}^{\infty}\right) .
$$

The set $\mathbf{H}^{\infty}$ is precisely the set of vertices representing simple closed curves whose homology class is nontrivial in $\Sigma$, but trivial in $H$. For a Heegaard splitting, it encodes homology information about the ambient manifold. In particular, the boundary distance determines precisely when a manifold has infinite homology (and therefore a nonseparating, incompressible surface). 
Lemma 4. The following are equivalent:

(1) the first homology group of $M$ is infinite;

(2) $M$ contains a nonseparating, two sided, closed incompressible surface;

(3) $d^{\infty}(\Sigma)=0$ and

(4) $d^{0, \infty}(\Sigma)=0$.

The proof is given in Section 2. The equivalence of (1) and (2) is well known, but we give a very simple, geometric proof via the boundary set. Theorem 1 is proved in Section 3.

For any Heegaard splitting $\left(\Sigma, H_{1}, H_{2}\right)$ of a non-Haken 3-manifold, Lemma 4 implies that the boundary set in $C(\Sigma)$ determined by $H_{2}$ must be completely disjoint from the boundary set for $H_{1}$. Hempel showed that there are handlebody sets that are arbitrarily far apart in the curve complex. The same is not true for boundary sets. In particular, Theorem 1 implies that for any Heegaard splitting $\left(\Sigma, H_{1}, H_{2}\right)$ of genus 3 or greater, $d^{\infty}(\Sigma)$ is equal to either 0,1 or 2 . For non-Haken manifolds, we have the following.

Corollary 5. For any Heegaard splitting $\left(\Sigma, H_{1}, H_{2}\right)$ of a non-Haken 3-manifold $M, d^{\infty}(\Sigma)$ is equal to 1 or 2 .

\section{Nonseparating surfaces}

The following Lemma will not be used until Section 3, but the method of proof gives a good introduction to the proof of Lemma 7. Recall that an element $\alpha$ of a $\mathbf{Z}$ module $G$ is called primitive if there is no $\beta \in G$ such that $\alpha=k \beta$ for some $k \neq \pm 1$.

Lemma 6. Let $\ell_{1}, \ldots, \ell_{k}$ be pairwise disjoint, essential loops in the boundary of a genus- $g$ handlebody $H$ with $g>k$. Then there is a properly embedded, nonseparating surface $F \subset H$ such that $\partial F$ is disjoint from each $\ell_{j}$ and the homology class defined by $\partial F$ in $H(\Sigma)$ is primitive.

Proof. Let $D_{1}, \ldots, D_{g}$ be a system of disks for $H$, that is, a collection of properly embedded, essential disks whose complement in $H$ is a single ball. Orient the boundaries of the disks and the loops $\ell_{1}, \ldots, \ell_{k}$, then form the matrix $A=\left(a_{i j}\right)$ such that $a_{i j}$ is the algebraic intersection number of $D_{i}$ and $\ell_{j}$.

If we replace one of the disks in the system by a disk slide, the matrix for the new system of disks can be constructed from $A$ by adding or subtracting one row from the other. Thus we can perform elementary row operations on $A$ by choosing new systems of disks for $H$. In particular, we can make $A$ upper triangular.

Because $A$ has more rows than columns, if $A$ is upper triangular then the bottom row consists of all zeros. In other words, the disk $D_{g}$ has algebraic intersection 0 with each loop $\ell_{j}$. 
If $D_{g}$ intersects the loop $\ell_{1}$, there must be a pair of adjacent intersections in $\ell_{1}$ with opposite orientations. By attaching a band from $\partial D_{g}$ to itself, along the arc of the loop $\ell_{1}$, we can form a new surface $F_{1}$ whose boundary (consisting of two loops) has algebraic intersection zero with each loop $\ell_{j}$, but whose geometric intersection number with the collection of curves $\ell_{1}, \ldots, \ell_{k}$ is strictly lower than that of $D_{g}$. The surface $F_{1}$ is two sided because the intersections have opposite orientations and nonseparating because $D_{g}$ is nonseparating.

If $\partial F_{1}$ intersects $\ell_{1}$, we can form a new surface $F_{2}$ by attaching a band, and so on. Continuing in this manner for each $\ell_{j}$, we form a surface $F$ which is properly embedded, two-sided, nonseparating and such that $\partial F$ is disjoint from each $\ell_{j}$.

Attaching a band to the boundary of $F_{i}$ does not change the homology class of the boundary, so the homology class of $\partial F$ is equal to the class of $\partial D_{g}$. Because $\partial D_{g}$ is represented by a connected loop, its homology class is primitive, as is the homology class of $\partial F$.

We will now use the idea of attaching bands to eliminate intersections to prove the implication $(1) \Rightarrow$ (4) of Lemma 4.

Lemma 7. If the first homology of $M$ is infinite then $d^{0, \infty}(\Sigma)=0$.

Proof. Let $D_{1}, \ldots, D_{g}$ be a system of disks for $H_{1}$ and $D_{1}^{\prime}, \ldots, D_{g}^{\prime}$ be a system of disk for $\mathrm{H}_{2}$. Orient the boundaries of both systems of disks. Let $A$ be the matrix of algebraic intersection numbers of the boundaries. Because the first homology is infinite, the determinant of $A$ must equal zero.

As in the last proof, we can perform row operations on $A$ by taking disk slides of the disks $D_{1}, \ldots, D_{g}$. Because the determinant of $A$ is zero, some sequence of disk slides will leave $A$ with all zeros in the bottom row. Thus after a sequence of disk slides, we can assume $D_{g}$ has algebraic intersection 0 with each $D_{j}^{\prime}$.

By attaching bands to the boundary of $D_{g}$ as in the proof of Lemma 6, we can form a properly embedded, two sided, nonseparating surface $F$ whose boundary is disjoint from $D_{1}^{\prime}, \ldots, D_{g}^{\prime}$. Thus each boundary component of $F$ bounds a disk in $H_{2}$. The union of $F$ and these disks is a properly embedded, two sided, nonseparating closed surface in $M$.

Recall that $F$ was constructed from $D_{g}$ by attaching bands to its boundary. The last band defines a boundary compression for $F$ corresponding to an isotopy pushing this last band into $\mathrm{H}_{2}$. After this isotopy, the second to last band defines a second isotopy, and so on. The final result is a surface isotopic to $F$ which intersects $H_{1}$ in a disk isotopic to $D_{g}$.

The intersection of this surface with $\mathrm{H}_{2}$ is orientable, two-sided and nonseparating because $F$ has these properties. Thus $\partial D_{g}$ is in both $\mathbf{H}_{1}^{0}$ and $\mathbf{H}_{2}^{\infty}$ so

$$
d^{0, \infty}(\Sigma)=d\left(\mathbf{H}_{1}^{0}, \mathbf{H}_{2}^{\infty}\right)=0 .
$$


Proof of Lemma 4. Lemma 7 implies that for any Heegaard splitting $\left(\Sigma, H_{1}, H_{2}\right)$,

$$
d\left(\mathbf{H}_{1}^{0}, \mathbf{H}_{2}^{\infty}\right)=0
$$

so (1) $\Rightarrow$ (4). Because $\mathbf{H}_{1}^{0}$ is contained in $\mathbf{H}_{1}^{\infty},(4) \Rightarrow$ (3) is immediate.

Let $\left(\Sigma, H_{1}, H_{2}\right)$ be a Heegaard splitting for $M$. If $d^{\infty}(\Sigma)=0$ then there is a simple closed curve $\ell \subset \Sigma$ such that $\ell$ bounds two-sided, nonseparating properly embedded surfaces $F \subset H_{1}$ and $F^{\prime} \subset H_{2}$. The union $F \cup F^{\prime}$ is a two-sided, nonseparating closed surface embedded in $M$. Compressing $F \cup F^{\prime}$ to either side produces at least one new two-sided, nonseparating surface. By compressing repeatedly, we eventually find a closed, nonseparating, two-sided incompressible surface in $M$. Thus (3) $\Rightarrow(2)$.

The final step, $(2) \Rightarrow(1)$, is a classical result. If $M$ contains a two-sided, nonseparating, closed surface $S \subset M$, let $p$ be a point in $S$. There is a path

$$
\alpha:[0,1] \rightarrow M
$$

from $p$ to itself that does not cross $S$. The homology class of $\alpha$ has infinite order so the first homology of $M$ is infinite.

\section{Density}

Proof of Theorem 1. We will prove the following: Let $\ell$ be a loop in $\partial H$ and assume the genus of $H$ is at least 3. Then there is an essential loop $\ell^{\prime}$ disjoint from $\ell$ and a properly embedded, two-sided, nonseparating surface $F$ such that $\partial F$ is a single, nonseparating loop disjoint from $\ell^{\prime}$.

By Lemma 6, there is a properly embedded surface $F^{\prime \prime} \subset H$ such that $\partial F^{\prime \prime}$ is disjoint from $\ell$ and defines a primitive element of the homology. Of all the properly embedded surfaces with boundary disjoint from $\ell$ and homologous to $\partial F^{\prime \prime}$, let $F^{\prime}$ be one with minimal number of boundary components. Each component of $\partial F^{\prime}$ has an orientation induced by $F^{\prime}$ and thus defines an element of the first homology of $\Sigma$.

For each component $C$ of $\Sigma \backslash\left(\ell \cup \partial F^{\prime}\right)$, an orientation for a loop in $\partial C$ induces an orientation of $C$. Assume two components of $\partial C$ come from loops of $\partial F^{\prime}$ and induce the same orientation of $C$. Because the induced orientations agree, adding a band between them produces a new orientable surface with fewer boundary components, but homologous boundary. Thus the minimality assumption implies that each component $C$ of $\Sigma \backslash\left(\ell \cup \partial F^{\prime}\right)$ has at most one boundary loop coming from $\partial F^{\prime}$ inducing each possible orientation. Thus it has at most two boundary loops coming from $\partial F^{\prime}$ and these induce opposite orientations on $C$.

Assume for contradiction each component of $\Sigma \backslash\left(\ell \cup \partial F^{\prime}\right)$ is planar. Each component that is disjoint from $\ell$ has exactly two boundary components. A planar 
surface with two boundary loops is an annulus so each component disjoint from $\ell$ must be an annulus. There are either two components of $\Sigma \backslash\left(\ell \cup \partial F^{\prime}\right)$ with one boundary loop each on $\ell$, or one component with two boundary loops on $\ell$. In the first case, the two components are pairs of pants or annuli, while in the second, the component is a four punctured sphere or a pair of pants. The union of such components and a collection of annuli is a genus-one or genus-two surface. This contradicts the assumption that $\Sigma$ has genus at least three, so we conclude that some component must be nonplanar.

Let $C$ be a nonplanar component. There is a simple closed curve $\ell^{\prime} \subset C$ such that $\ell^{\prime}$ separates a once-punctured torus from $C$. In $\Sigma$, the loop $\ell^{\prime}$ separates a oncepunctured torus that contains no components of $\partial F^{\prime}$. Let $F$ be a surface whose boundary is homologous to $F^{\prime}$, disjoint from the once-punctured torus bounded by $\ell^{\prime}$ and such that the number of boundary components of $F^{\prime}$ is minimal over all such surfaces.

Once again, each component of $\Sigma \backslash\left(\ell^{\prime} \cup \partial F\right)$ has at most two boundary components on loops in $\partial F$, with opposite induced orientations. Because $\ell^{\prime}$ bounds a surface disjoint from $\partial F$, each component of $\Sigma \backslash \partial F$ must also have at most two boundary loops on $\partial F$.

If a component $C$ of $\Sigma \backslash \partial F$ has a single boundary component, this loop is homology trivial in $\Sigma$. Attaching a boundary parallel surface to $F$ removes this loop so minimality of $\partial F$ implies that each component has two boundary loops.

If $C$ has two boundary loops (with opposite induced orientations) then these loops determine the same element of the homology of $\Sigma$. Because $\Sigma$ is connected, this implies that any two loops of $\partial F$ (with their induced orientations) determine the same element of the homology. Thus the element of the homology determined by $\partial F$ is of the form $k \beta$ where $k$ is the number of boundary components of $F$.

By Lemma 6, $\partial F$ determines a primitive element of the homology of $\Sigma$, so $k$ must be 1 . In other words, the boundary of $F$ is connected and $\partial F$ determines an element of $\mathbf{H}^{\infty}$. By construction, $\partial F$ is disjoint from a loop $\ell^{\prime}$ that is disjoint from $\ell$. Thus the vertex $v \in C(\Sigma)$ determined by $\ell$ is distance at most 2 from $\mathbf{H}^{\infty}$.

\section{References}

[Hempel 2001] J. Hempel, "3-manifolds as viewed from the curve complex", Topology 40:3 (2001), 631-657. MR 2002f:57044 Zbl 0985.57014

[Jaco 1980] W. Jaco, Lectures on three-manifold topology, CBMS Regional Conference Series in Mathematics 43, American Mathematical Society, Providence, R.I., 1980. MR 81k:57009 Zbl 0433.57001

[Scharlemann 2006] M. Scharlemann, "Proximity in the curve complex: boundary reduction and bicompressible surfaces”, Pacific J. Math. 228:2 (2006), 325-348. MR 2008c:57035 Zbl 05190398 [Schleimer 2005] S. Schleimer, "Notes on the complex of curves", lecture notes, 2005, Available at http://www.math.rutgers.edu/ saulsch/math.html. 
Received July 11, 2007. Revised September 27, 2007.

JESSE EDWARD JOHNSON

MATHEMATICS DEPARTMENT

YALE UNIVERSITY

PO BOX 208283

NEW HAVEN, CT 06520-8283

UNITED STATES

jessee.johnson@yale.edu

http://math.yale.edu/ jj327/

TERK PATEL

18 Dumas STREeT

PONDICHERRY - 605001

INDIA

terkpatel@yahoo.com 